\title{
Punto interdomal en técnica cerrada
}

\section{Interdomal suture in closed approach}

\author{
Roberto Arias A¹, Leandro Rodríguez $\mathrm{H}^{1}$, Mónica Rojas $\mathbf{0}^{2}$, Mariana Bogado C1.
}

\begin{abstract}
RESUMEN
El manejo de la punta nasal es un elemento clave en toda rinoplastía. Las primeras técnicas empleadas sacrificaban la estructura del arco alar con resultados posoperatorios indeseables. En la era moderna de la rinoplastía, el manejo de la punta nasal se enfocó en remodelar, preservar y reposicionar los cartílagos alares mediante el uso de suturas. El punto interdomal es una sutura en forma de ocho o loop simple desde la porción más anterior de un domo hacia el contralateral uniendo la región más alta de las cruras mediales. En el presente artículo se realiza una revisión del uso de puntos de suturas en el manejo de la punta nasal, se describen las características del punto interdomal en técnica cerrada y se muestran sus resultados en una serie de casos.
\end{abstract}

Palabras clave: Punta nasal, puntos de sutura, punto interdomal en técnica cerrada.

\begin{abstract}
The nasal tip surgery is a key element in rhinoplasty. The first techniques employed sacrificed the alar arch structure with undesirable postoperative results. In the modern era of rhinoplasty, nasal tip surgery focused on reshaping, preserving and repositioning the alar cartilages using reversible sutures. The interdomal suture is a figure-eight or simple loop suture placed from the most anterior portion of one dome to the contralateral joining the highest region of the medial crura. This article reviews the use of suture techniques in nasal tip reshaping and describes the interdomal suture in closed approach and its results in a clinical series.
\end{abstract}

Key words: Nasal tip, suture techniques, interdomal suture in closed approach.

\footnotetext{
${ }^{1}$ Médicos Servicio de Otorrinolaringología, Hospital del Salvador.

${ }_{2}^{2}$ Médico Cirujano Hospital del Salvador.
} 


\section{INTRODUCCIÓN}

El manejo de la punta nasal siempre ha sido considerado un elemento clave para una rinoplastía exitosa, siendo sus objetivos principales crear una punta más angosta y más definida ${ }^{1-3}$. Ya a inicios del siglo XX Fomon declaró "quien domina la punta nasal, domina la rinoplastía", afirmación que sigue siendo válida hoy en día ${ }^{4}$.

Lograr un aspecto natural de la punta nasal es una tarea compleja y requiere que el cirujano realice un enfoque tridimensional de la anatomía de la nariz, teniendo siempre en consideración la forma y posición de la punta nasal, el grosor y tipo de piel, la calidad de los cartílagos y la etnia del paciente ${ }^{5}$.

\section{HISTORIA}

Las primeras técnicas en rinoplastía empleadas para el manejo de la punta nasal se centraban en un principio básico: Sacrificar la integridad de la crura lateral para aumentar la crura medial y así ganar proyección y altura en la punta. No obstante, el problema principal de estas técnicas con resección excesiva de cartílago es su irreversibilidad al perturbar la integridad estructural del arco alar. Además los resultados posoperatorios suelen ser indeseables y poco predecibles, ya que reducen el apoyo intrínseco de la punta nasal, disminuyen la proyección de la punta, debilitan los cartílagos alares y favorecen la retracción cicatricial ${ }^{1,4,6,7}$.

Es por esto que en la era moderna de la rinoplastía se dio paso a un cambio en el manejo de la punta nasal. Su filosofía se centra en la remodelación, preservación y reposición de los cartílagos alares existentes mediante el uso de suturas sin necesidad de resecar tejido $0^{6-9}$ en pacientes debidamente seleccionados.

Dentro de las ventajas que tiene el uso de puntos de suturas para modificar la punta nasal, por sobre las técnicas antes empleadas, destaca su carácter reversible, el ser técnicas que preservan la anatomía y estructuras del arco alar, que permiten aumentar los mecanismos de soporte de la punta nasal y que además presentan resultados posoperatorios más predecibles basados en el sitio de localización de la sutura ${ }^{9,10}$.
Actualmente existen diversos puntos de sutura para modificar la punta nasal entre los cuales se encuentra la sutura Interdomal6,10.

\section{DOMOS NASALES}

Los domos nasales corresponden a la zona de transición entre la crura medial y la crura lateral conformando la región más proyectada de la punta nasal.

\section{MEDIDAS ANATÓMICAS DE LA PUNTA NASAL}

Algunas medidas que debemos conocer para manejar la punta nasal son: La distancia interdomal que no debe superar los 8 a $10 \mathrm{~mm}$ para que la punta no pierda definición. Esta distancia está regida por el ángulo de divergencia interdomal que no debe ser mayor a $30^{011}$. El ángulo de definición domal debe ser cercano a los $90^{\circ}$ y el arco domal debe ser igual o menor de $4 \mathrm{~mm}^{1,11}$ (Figura 1).

\section{PUNTO INTERDOMAL CLÁSICO}

Jaques Joseph, el padre de la rinoplastía moderna, describió este punto por primera vez en $1931^{6,13}$. Este punto consiste en una sutura en forma de 8 o loop simple que va desde la porción

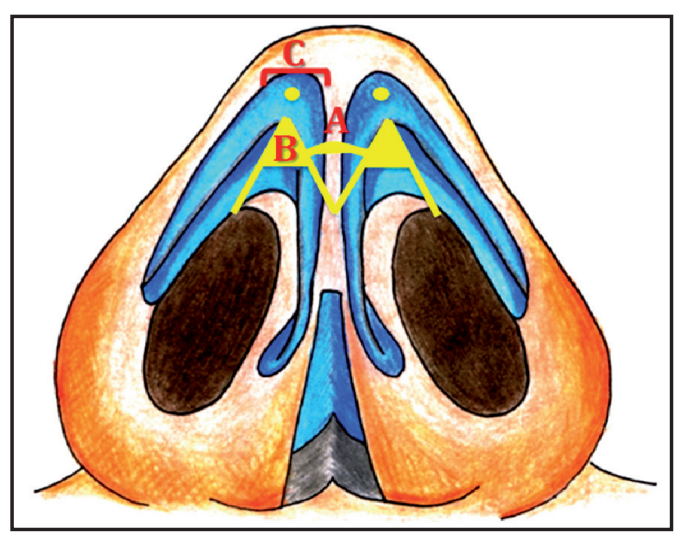

Figura 1. A: ángulo de divergencia interdomal $\leq 30^{\circ}$. B: ángulo

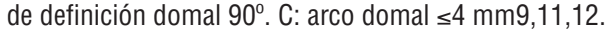


más anterior de un domo hacia el domo contralateral, uniendo la parte más alta de las cruras mediales ${ }^{10,14}$

De acuerdo a diversos autores, los objetivos que busca alcanzar el punto interdomal son acortar la distancia entre los domos, angostar la punta, aumentar el largo y volumen de los lóbulos y mejorar la proyección de la punta nasal|,10,14-16. Su uso estaría indicado en casos donde existe un aumento en el ángulo de divergencia interdomal, pero con una buena conservación de la anatomía de los domos ${ }^{10}$.

\section{DESCRIPCIÓN DE LA VARIANTE DEL PUNTO INTERDOMAL PARA TÉCNICA CERRADA}

Para el abordaje cerrado el punto interdomal consiste en pasar una sutura a través de la región cefálica del cartílago alar inferior a 3 ó $4 \mathrm{~mm}$. por sobre el borde superior de la incisión caudal transfixiante del septum nasal en la región supradomal (Figuras 2, 3 y 4).

Si la punta nasal es muy ancha se colocará otro punto en el mismo borde cefálico de la crura lateral a $8 \mathrm{~mm}$, en la región supradomal, a partir del borde superior de la incisión caudal lográndose con esto disminuir aún más el ancho de la punta nasal (Figura 5).

Generalmente se coloca un punto a $4 \mathrm{~mm}$ y otro a $8 \mathrm{~mm}$ siempre considerando las características de cada nariz, por esto debe presentarse la sutura para ver cuánto se angosta el domo previo a su ubicación definitiva (Figuras 6 y 7).

Posteriormente se anuda el punto en la región central aproximando ambos bordes, lográndose así disminuir la distancia interdomal (Figuras 7 y 8). Luego el punto que está a $4 \mathrm{~mm}$ se sutura y se corta. Posteriormente el que está a $8 \mathrm{~mm}$ se sutura y se tracciona fijándolo al septum cartilaginoso en su borde dorsal con lo cual se logra proyectar la punta nasal en la posición que queremos (Figura 9).

Siempre debemos considerar el uso de técnicas auxiliares que permitan mantener los cambios como la sutura del septum a las cruras medias y, aquellos pacientes que necesitan mayores cambios, puede dejarse un poste evitando la desproyección nasal a largo plazo.

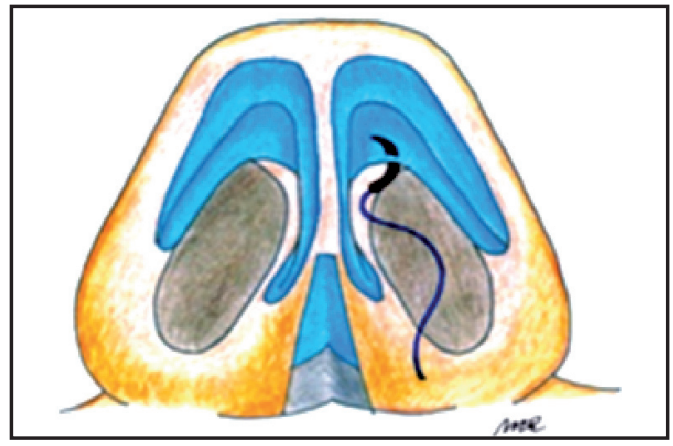

Figura 2.

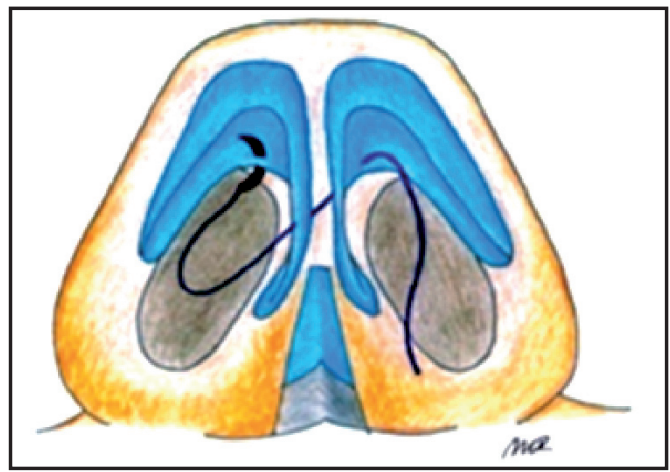

Figura 3.

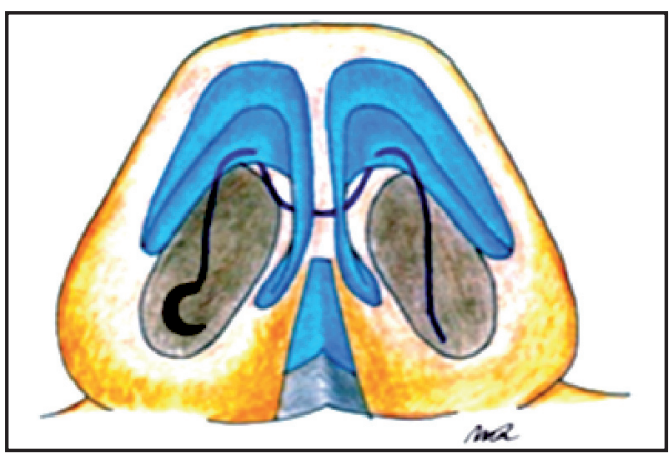

Figura 4.

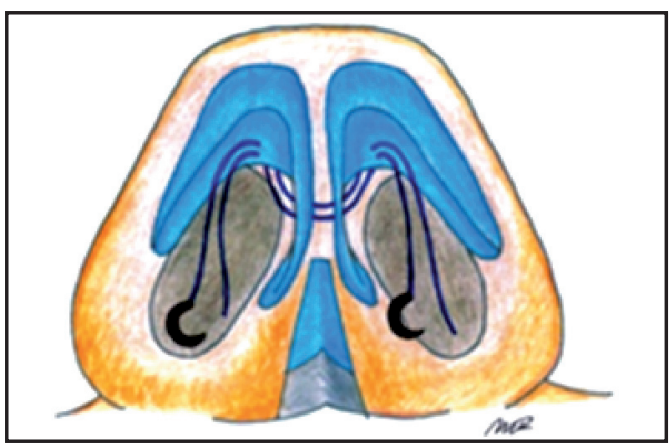

Figura 5. 
El punto interdomal debe realizarse dos veces, especialmente en narices globosas, para asegurar el resultado y prevenir la deformación que podría producirse frente a un desgarro de los cartílagos.

\section{EXPERIENCIA CLÍNICA}

En un período de 3 años, a 102 pacientes se les realizó una rinoplastía con abordaje cerrado y con

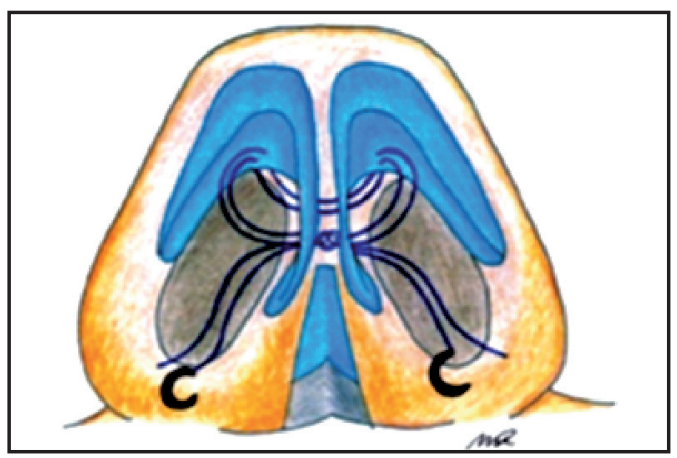

Figura 6.

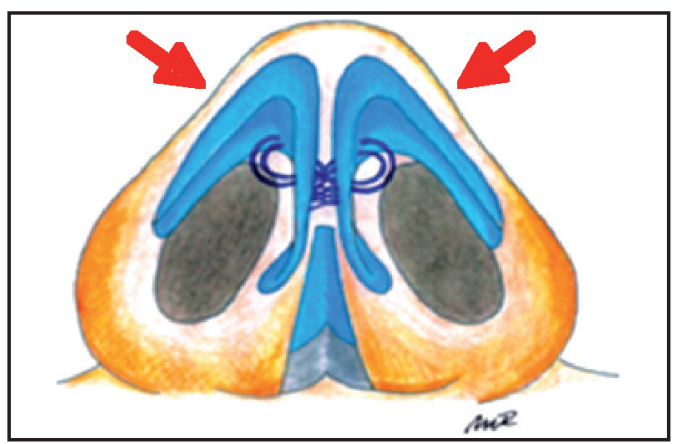

Figura 7.

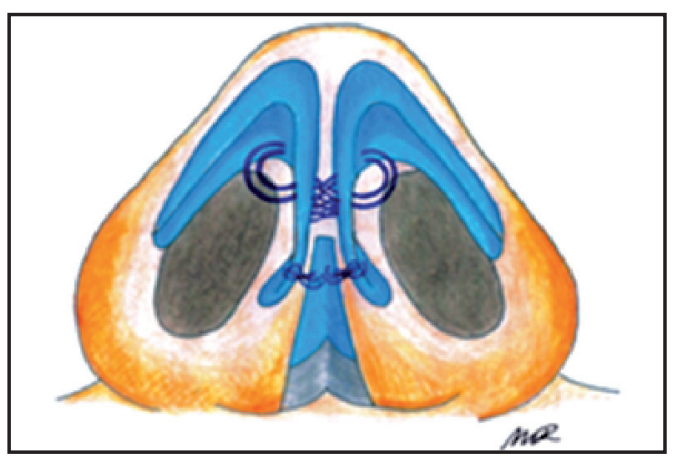

Figura 8. manejo de la punta nasal mediante el uso del punto interdomal modificado anclado al septum nasal.

Los pacientes seleccionados para este tipo de cirugía fueron elegidos por el cirujano cumpliendo dos características principales: no presentar grandes alteraciones anatómicas y tener una punta nasal desproyectada.

Definir con más detalle y exactitud el tipo de paciente seleccionado es difícil, ya que la rinoplastía lleva implícito un tercio de habilidad, un tercio de conocimiento y un tercio de arte siendo este último un aspecto subjetivo que se adquiere y maneja con la experiencia y teniendo como objetivo final la armonía facial.

Sabemos que no es posible solucionar todas las deformaciones nasales con un solo punto de sutura, por esto siempre que fue necesario se complementó con técnicas coadyuvantes para lograr y mantener los cambios de las estructuras modificadas. Por ejemplo resecar el borde cefálico del cartílago alar, tira superior del cartílago cuadrangular, punto de fijación del septum nasal a las cruras mediales o la colocación de un vástago en la columela. Este último se posiciona por vía cerrada mediante un bolsillo retrocolumelar en pacientes con puntas nasales muy desproyectadas.

Con la variante de la sutura interdomal descrita se consiguió, en la mayoría de los casos, acortar

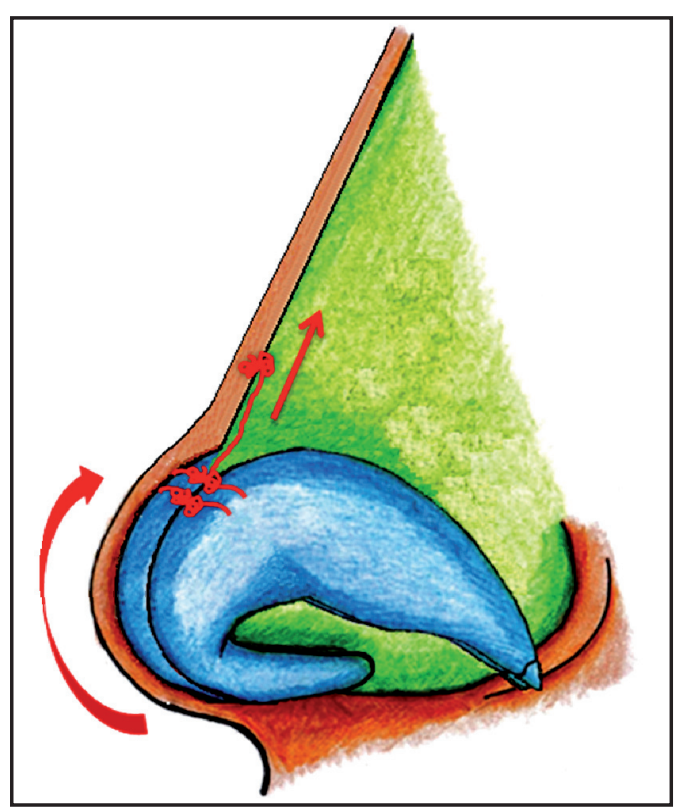

Figura 9. 


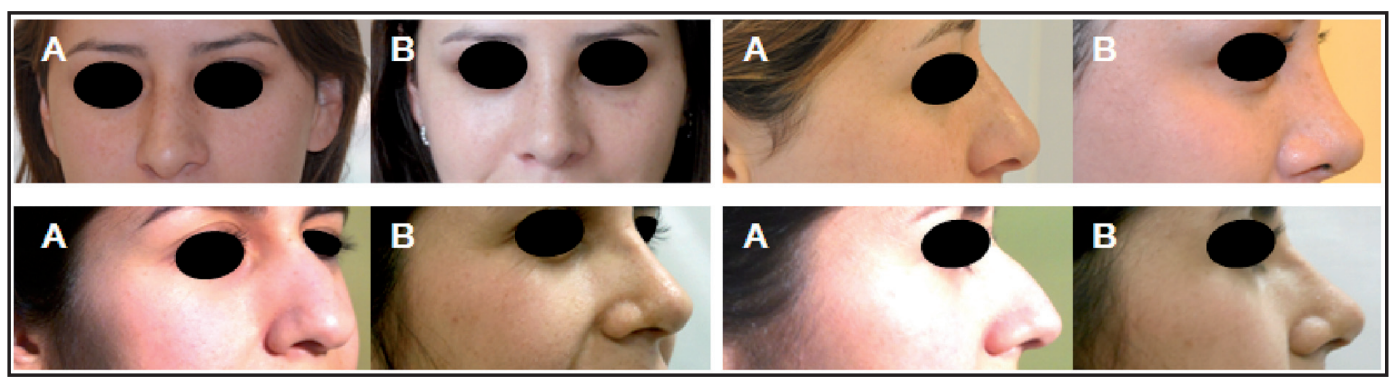

Figura 10. Fotografías del pre (A) y posoperatorio (B) de pacientes en quienes se modificó la punta nasal mediante la variante del punto interdomal.

la distancia interdomal para disminuir el ancho nasal y mejorar la proyección de la punta nasal, fundamentalmente gracias a la sutura al septum nasal (Figura 10).

\section{DISCUSIÓN}

Hoy en día el uso de puntos de sutura reversibles para el manejo de la punta nasal es una alternativa real a las técnicas resectivas inicialmente empleadas. Su beneficio reside en que permiten modificar la punta nasal sin perturbar las estructuras de soporte de la nariz, alcanzando resultados posoperatorios más predecibles y permanentes en el tiempo.

La variante del punto interdomal para técnica cerrada permite disminuir la distancia entre los domos, angostar y mejorar la proyección de la punta nasal, alcanzando objetivos similares a los descritos para la sutura en el abordaje abierto. Al realizar puntos de fijación al septum cartilaginoso se logra proyectar mejor la punta nasal, permitiendo mantener las modificaciones realizadas en el tiempo. Además al efectuar dos puntos interdomales contiguos, en vez de uno sólo, se disminuye la probabilidad de desgarro de los cartílagos alares, siendo esto fundamental para mantener la nueva estructura de los domos a largo plazo.

\section{CONCLUSIÓN}

Toda rinoplastía que requiera del manejo de la punta nasal debe tener una evaluación preoperatoria exhaustiva para definir el tipo de técnica y sutura a emplear. Mediante la técnica quirúrgica elegida, idealmente se deben modificar y reposicionar los cartílagos alares resecando la menor cantidad de tejido y así preservar las estructuras de soporte de la punta nasal.

Con la variante descrita del punto interdomal para técnica cerrada se logra un aspecto natural de la nariz, con una buena proyección y rotación de la punta nasal, pero sin alterar la estabilidad del arco alar.

\section{BIBLIOGRAFÍA}

1. ToRiumi DM. New Concepts in Nasal Tip Contouring. Arch Facial Plast Surg 2006; 8(3): 156-85.

2. Cárdenas L, Guerrero M. Improving Nasal Tip Projection and Definition Using interdomal Sutures and Open Approach without Transcolumellar Incision. Aesth Plast Surg 2002; 26: 161-6.

3. Eisenmann-Klein M, Neuhann-Lorenz C. Innovations in Plastic and Aesthetic Surgery 2008. Chapter 34: Refinements in Nasal Tip Surgery, págs. 296-300.

4. VuYk HD. Suture Tip Plasty. Rhinology 1995; 33(1): 30-8.

5. Pedroza F. A 20-Year Review of the New Domes Technique for Refining the Drooping Nasal Tip. Arch Facial Plast Surg 2002; vol 4: 157-63.

6. RA, Ghavami A, Guyuron B. Nasal Tip Sutures Part I: The Evolution. Behmand Plast Reconstr Surg 2003; 112(4): 1125-9; discussion 1146-9.

7. Nassif Filho A, Romano G, Braga D, Gambeta $S$, Franceschi E. Nasal Tip Narrowing: Minimally Invasive Suture Technique for Thick Nose Tip. 
Intl Arch Otorhinolaryngol 2011; vol 15, $\mathrm{n}^{0} 3$ : 302-7.

8. Corrado A, Bloom J, Becker D. Domal Stabilization Suture in Tip Rhinoplasty. Arch Facial Plast Surg 2009; 11(3): 194-7.

9. LeACH JL, Athré RS. Four Suture Tip Rhinoplasty: A Powerful Tool For Controlling Tip Dynamics. Otolaryngol Head Neck Surg 2006; 135(2): 22731.

10. Guyuron B, Behmand RA. Nasal Tip Sutures Part II: The Interplays. Plast Reconstr Surg 2003; 112(4): 1130-45; discussion 1146-9.

11. Rohrich R, Adams W JR. The Boxy Nasal Tip: Classification and Management Based on Alar Cartilage Suturing Techniques. Plast Reconstr Surg 2001; 107(7): 1849-63; discussion 1864-8.
12. Pasinato R, Mocelin M, Sarraf C. Nose Tip Refinement Using Interdomal Suture in Caucasian Nose. Int Arch Otorhinolaryngol 2012; 16(3): 391-5.

13. Emmerich M, Emmerich E. Historia de la Rinoplastía Estética. Rev Otorrinolaringol Cir Cabeza Cuello 2001; 61: vol 3.

14. Guyuron B. Bahman Guyuron Rhinoplasty 2012. Chapter 6 Tip Suture: Interdomal Suture, pág. 143.

15. Gruber R, Chang E, Buchanan E. Suture Techniques in Rhinoplasty. Clin Plast Surg 2010; 37(2): 231-43.

16. Ghavami A, Janis J, Acikel $C$, Rohrich R. Tip Shaping in Primary Rhinoplasty: An Algorithmic Approach. Plast Reconstr Surg 2008; 122(4): 1229-41.

Dirección: Mónica Rojas 0.

Hospital del Salvador

E mail: mrojas.olavarria@gmail.com 